\title{
Originales
}

\section{Optimización de la técnica radiográfica del tórax PA}

\author{
Patricia-Mora'
}

J ustificación y objetivo: En Costa Rica las placas PA de tórax se toman con kilovoltajes bajos ( $<100 \mathrm{kV}$ ), en contraposición con las recomendaciones internacionales. El estudio tiene como objetivo demostrar que al aumentar el kilovoltaje, la visualización de los datos diagnósticos de objetos de bajo contraste mejora y las dosis entregadas disminuyen.

\begin{abstract}
M étodos: A un total de 11 pacientes se les tomó una placa de alto $\mathrm{kV}$ y y otra a bajo kV p. Sobre la piel del paciente se colocaron 2 cristales TLD-100 para cuantificar las dosis de entrada, que posteriormente fueron analizados en la Sección de Dosimetría del Laboratorio de Física Nuclear de la U niversidad de Costa Rica. U tilizando el criterio anatómico, 28 médicos radiólogos compararon los hallazgos radiológicos para ambas técnicas.
\end{abstract}

Resultados: A I aumentar el kilovoltaje se encontró que las dosis promedio de entrada a la piel disminuyerón en un $40 \%$ y el contraste de las imágenes, disminuyó entre un $10 \%$ y un $50 \%$. La sumatoria general para el análisis del criterio anatómico fue de 448 puntos positivos, lo que indica que el grupo de médicos visualizó mejor las estructuras anatómicas con la técnica del al to kilovoltaje.

Conclusiones: La optimización del estudio del tórax con base en la razón beneficio versus riesgo radiológico, señala que la técnica del alto $\mathrm{kV} p$ deberá ser implementada en nuestro país, pues disminuye la dosis de radiación entregada al paciente, objetivo primordial dentro de la protección radiológica, y permite una mejor visualización de la anatomía del área estudiada, por parte del médico.

Descriptores: radiología, tórax, dosis, contraste.

Recibido: 28 de junio, 2000

Aceptado: 08 de octubre, 2002.

En los servicios de radiodiagnóstico de Costa Rica, así como en el resto del mundo, las radiografías de tórax son los estudios más comunes. 1,2,3 Sin embargo, obtener una radiografía de tórax óptima para diagnóstico no es sencillo, debido a su compleja anatomía y a las variadas condiciones patológicas. EI porcentaje de retomas llega a ser hasta de un $10 \%$. 4.

Una adecuada placa (PA) de tórax tiene que permitir la completa visualización de ambos pulmones, las escápulas no se deben superponer al campo pulmonar, los primeros tres cuerpos vertebral es deben ser apenas visibles a través de la silueta cardíaca, las puntas de las clavículas deben estar equidistantes de la línea media y entre otras cosas, deberá tomarse en inspiración máxima, con el paciente en posición de pie. ${ }^{6}$

A breviaturas: $\bar{T} \bar{L} \bar{D}$; dosimetría termoluminiscente; ICRP; Comisión Internacional de Protección Radiológica; PA; posteroanterior; mGy; miligray; D O; densidad óptica; kV p; kilovoltaje pico; mSv; milisievert.

1 Laboratorio de Física Nuclear A plicada, Escuela de Física, Universidad de Costa Rica, San J osé, Costa Rica.
El uso efectivo de cualquier modalidad de imagenología diagnóstica y la interpretación de sus imágenes requiere de un conocimiento de los principios físicos del proceso de formación de la imagen. Lo anterior debido a que la habilidad para visualizar una estructura anatómica específica o una condición patológica, depende de las características inherentes de la modalidad diagnóstica y de un grupo de factores externos seleccionados por el operador. La relación entre la visibilidad y estos factores es compleja, y generalmente conlleva compromisos, algunos que afectan incluso la calidad final de la imagen. Para agravar la situación, los métodos diagnósticos depositan energía en el cuerpo del paciente, lo que implica un posible riesgo biológico. La cantidad de dosis suministrada por los Rayos X diagnósticos afectará la calidad de la imagen final que recibe el radiólogo. Un procedimiento de imágenes optimizado es aquel en el cual estos dos factores, calidad final de la imagen y dosis entregada, están correctamente balanceados. ${ }^{7}$

L a combinación pantalla-película ha sido tradicionalmente el método estándar para la obtención de la radiografía del tórax, dada a su simplicidad, bajo costo, excelente resolución 
espacial y sensibilidad; pero tiene el inconveniente de que su habilidad es limitada para proveer el contraste deseado entre los pulmones y el mediastino. ${ }^{8}$

Para que un objeto sea visible en una imagen de Rayos $X$, debe tener contraste físico que lo diferencie de los tejidos adyacentes, el Ilamado contraste del objeto. Este se puede deber a diferencias en densidades, composición química (número atómico) o grosor del obj eto. Al irradiar el obj eto de interés y tomar en cuenta las características propias del haz de radiación (kilovoltaje, ánodo y filtro), el contraste se denomina contraste del sujeto. Finalmente, al incorporar las características del sistema receptor (tipo de película, líquidos reveladores y técnicas radiográficas) tenemos el contraste final de un objeto y su entorno. '

El tórax es una zona de muy alto contraste por tener áreas muy blancas (mediastino) y muy negras (pulmón), y por lo tanto la visualización de objetos de interés se dificulta en estas regiones. Se encuentra documentado que las películas radiográficas pierden la capacidad de demostrar el contraste de los objetos si estos se encuentran localizados sobre una zona muy blanca o muy negra de la película. 7,8,9

A sí, para visualizar objetos de bajo contraste en una placa de tórax, se recomienda disminuir el contraste a la imagen final. El contraste se puede reducir al implementar alguna de las siguientes acciones: utilizar una película de latitud, ${ }^{8}$ usar filtros compensadores entre la salida del haz y el paciente, ${ }^{10} \mathrm{y}$, finalmente, emplear un haz muy penetrante, o sea, con alto kilovoltaje. A menor kilovoltaje, se produce mayor efecto fotoeléctrico en los tejidos blandos, lo que da como consecuencia un mayor contraste (tal es el caso de la mamografía). Al aumentar el kilovoltaje, disminuye el efecto fotoeléctrico y aumenta el efecto Compton, y disminuye también el contraste de la imagen final. ${ }^{7} \mathrm{~L} 0$ anterior se muestra en las imágenes de la Figura N 0.1

Una investigación realizada en pacientes del Hospital Calderón Guardia de Costa Rica ${ }^{11}$, demostró que en los estudios de tórax se estaban entregando dosis mayores a las recomendadas internacionalmente. ${ }^{12,13,14} \mathrm{~L}$ a razón primordial obedece a los bajos kilovol tajes empleados (60-90 kV p), en contraposición con las recomendaciones internacionales de usar kilovoltajes entre 125-150 kV p. 2,8,9,12

Este trabajo tiene como objetivo demostrar al cuerpo médico nacional, que por medio de imágenes menos contrastadas del tórax (de alto kilovoltaje), la visualización de las estructuras anatómicas mejora y se logra disminuir las dosis entregadas a los pacientes.

\section{Materiales y métodos}

A 11 pacientes del Hospital Dr. Rafael Ángel Calderón Guardia, se les realizó una teleradiografía de tórax (PA) con técnica de alto kilovol taje (de 125 a 133 kV p dependiendo de la contextura del paciente con 1 ó $2 \mathrm{mAs}$ y a $1.8 \mathrm{~m}$ de

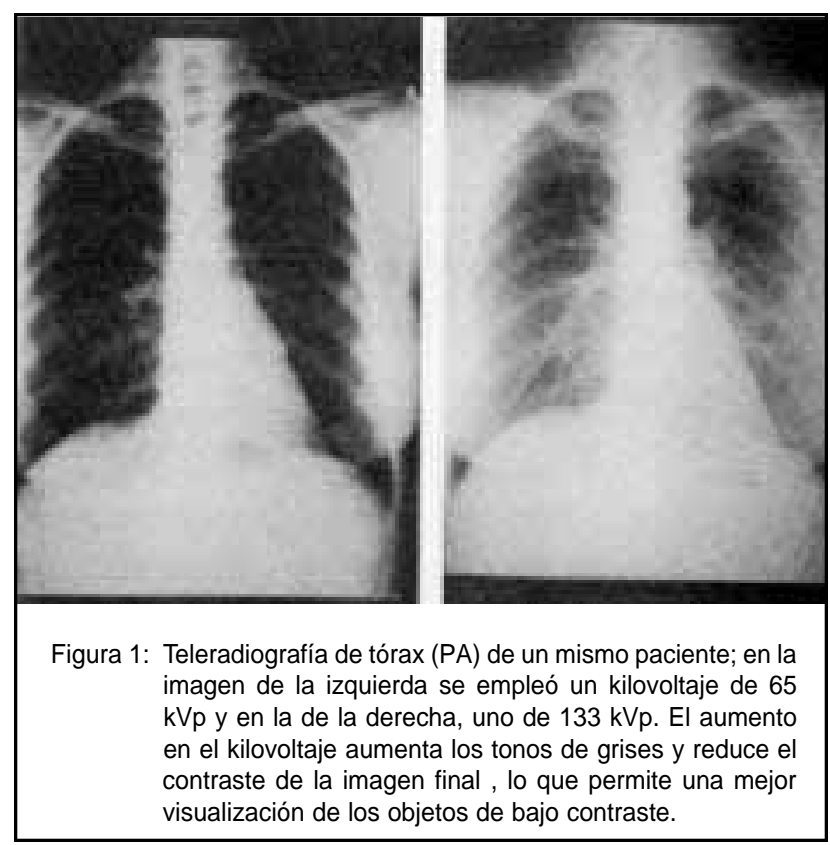

distancia del tubo), y otra a bajo kilovoltaje (de 65 a $75 \mathrm{kV} \mathrm{p}$ dependiendo de la contextura del paciente con 16 ó $20 \mathrm{mAs}$ y a $1.8 \mathrm{~m}$ de distancia del tubo), ambas con los mismos insumos radiológicos (películas, casetes y líquidos reveladores). Sobre la piel del paciente se coloco 2 cristales termoluminiscentes (TLD-100: LiF) para determinar las dosis de entrada a piel ${ }^{15,16}$ en cada técnica, con el propósito de cuantificar la disminución de la dosis al utilizar la técnica de alto kilovoltaje. Los cristales fueron leídos en la Sección de Dosimetría y Radioprotección del Laboratorio de Física Nuclear A plicada de la U niversidad de Costa Rica, empleando la metodología estándar. ${ }^{11}$

Para cuantificar en la disminución del contraste radiológico entre las 2 técnicas se utiliza un densitómetro (X-RITE Modelo: 331 con incertidumbre de $\pm 0.1 \mathrm{DO})$, que mide la diferencia en las densidades ópticas entre la base de la silueta cardíaca del lado izquierdo y el ángulo costo diafragmático izquierdo, sin superposición costal. ${ }^{9}$

Con el fin de comparar la información diagnóstica de ambas técnicas radiográficas, se elaboró un cuestionario basado en la metodología del criterio anatómico, ${ }^{5,17}$ en donde los diferentes hal lazgos radiológicos y al gunas patologías importantes de la región se comparan cualitativamente. EI médico radiól ogo analizó ambas placas de forma simultánea, colocando siempre la de mayor kilovoltaje a la izquierda del negatosco pio, y para los hallazgos radiológicos o patologías que se encuentran en el cuestionario procedió a asignarles una puntuación: dos puntos positivos (+2) si lo que se evaluaba "se observaba mucho mejor en la placa de al to kV p"; un punto positivo (+1) si "se observaba mejor en la placa de alto kVp"; cero puntos (0) si "se observaba igual en ambas placas"; un punto negativo (-1) si "se observaba mejor en la placa de bajo kVp" y dos puntos negativos (-2) si 
"se observaba mucho mejor en la placa de bajo kV p". Si consideró que el hallazgo radiológico o patología en cuestión no se podía valorar para determinado paciente, lo calificó como NA (no aplica), ya que las placas pueden corresponder a pacientes sanos. Se adoptó el del método de análisis estadístico multivariable de correlación cruzada para obtener de los resultados de las encuestas. ${ }^{18}$ De esta manera, un resultado neto final positivo favorece la técnica propuesta en la investigación (altos kilovoltajes). El "criterio anatómico" como herramienta metodológica se ha utilizado regularmente para complementar las mediciones físicas realizadas cuando los sistemas radiográficos son modificados (en este caso, el uso de 2 kilovoltajes diferentes), ya que disminuye la subjetividad del médico y permite analizar sus evaluaciones. Se basa en que si la visualización de un hallazgo anatómico se mejora al cambiar el tipo de estudio, se espera que la del hallazgo patológico también lo haga.

Las placas de los 11 pacientes fueron analizadas por 28 médicos, 18 radiólogos (64\%) y 10 residentes (36\%) del postgrado de Radiología e Imágenes M édicas, durante el período comprendido entre el 16 de junio y el 15 de noviembre de 2001. Los años de experiencia oscilaban desde residentes de primer año hasta médicos con 35 años de práctica en ese campo; la experiencia promedio de los médicos consultados era de al rededor del 10.5 años.

Los pacientes que participaron en el estudio fueron 9 hombres y 2 mujeres, los cuales podían estar sanos o enfermos en el momento de tomarles la radiografía, y su selección se basó en que no tuvieran evidente sobrepeso ni deformidad torácica, y que su rango de edad oscilara entre los 45 y 60 años. Todos aceptaron participar voluntariamente, bajo consentimiento informado en el protocolo descrito en el proyecto de investigación de la Vicerrectoría de Investigación de la Universidad de Costa Rica, N 0.112-A 0-003: "Optimización de las Técnicas Radiográficas del Tórax".

\section{Resultados}

En la Figura 2 se muestran los resultados encontrados por los dosímetros termoluminiscentes de la dosis de entrada a piel (mGy) recibida por cada paciente, para ambos kilovoltajes. La dosis promedio para la técnica de alto kilovoltaje es de $0.29 \mathrm{mG}$ y y el rango de variación es de 0.21 a $0.4 \mathrm{mG}$. La dosis promedio para la técnica de bajo kilovoltaje es de 0.47 $\mathrm{mG}$ y y el rango de variación es de 0.26 a $0.64 \mathrm{mG}$ y. El valor recomendado por organismos internacionales como aceptable en radiografía diagnóstica para un paciente adulto típico (conocida como nivel orientativo de dosis en el marco de la protección radiológica), en un tórax PA es de 0.4 mG y y se indica en la figura por la línea punteada. ${ }^{14}$ En el paciente N 0.9, la dosis para la placa de alto kilovoltaje no se pudo cuantificar por problemas en la Lectora Harshaw 4000.

Las mediciones del contraste radiológico de las dos placas para cada paciente, mostraron una disminución promedio en el contraste de un 31\%, con variaciones individuales para los

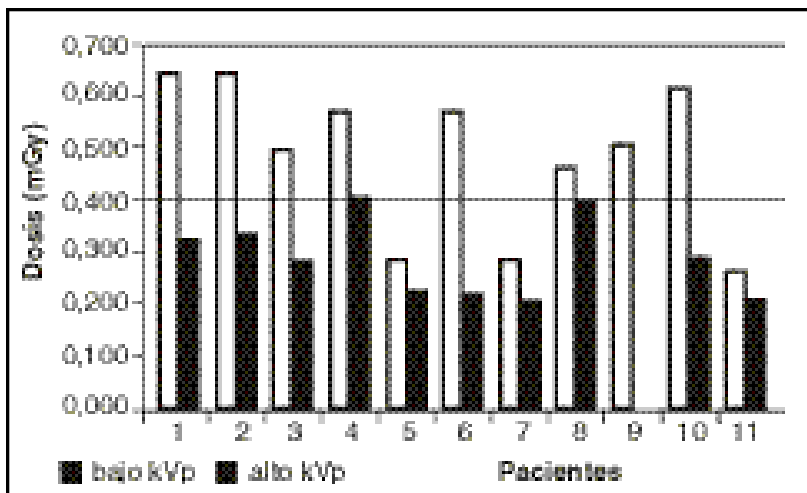

Figura 2: Dosis de entrada a piel en mGy para cada uno de los pacientes con baja y alta técnica. La línea corresponde al valor recomendado internacionalmente para un tórax PA. Todas las dosis entregadas con la técnica de alto kilovoltaje se encuentran por debajo del valor recomendado, no así para las dosis con las técnicas de bajo kilovoltaje. La dosis del paciente 9 para alto kilovoltaje no se pudo cuantificar.

diferentes pacientes desde un 10\% hasta un 50\%. Esta disminución en el contraste, debida al aumento de la energía del haz, la visualiza el médico como una imagen con más tonos de grises, como se evidencia en la Figura N o.1.

El análisis estadístico multivariable de correlación cruzada, aplicado a las 308 muestras (28 médicos por 11 pacientes), revela la frecuencia de preferencia para cada hallazgo radiológico, con base en la puntuación del criterio anatómico aplicado. Estos resultados se encuentran tabulados en el Cuadro N 0.1. La sumatoria general de todas las entradas del Cuadro No.1 (para todos los criterios de cada uno de los hallazgos) tiene un valor de 448 puntos positivos.

Así mismo, este análisis estadístico permitió encontrar la preferencia por alguna de las dos técnicas estudiadas con respecto a: los pacientes, los gustos del médico y la estructura anatómica analizada, de la siguiente manera:

10 de los 11 pacientes recibieron un puntaje total positivo, Io que indica que fueron mejor evaluados con la técnica de alto kilovoltaje. El único que obtuvo calificación negativa (mejor en la placa de bajo kVp) presentaba radiografías muy similares visual mente, para ambas técnicas radiográficas.

Un 54\% de los médicos prefirieron la técnica de al to kilovoltaje, ya que su puntuación resultó una cantidad positiva.

Los porcentajes absolutos de la frecuencia de preferencia para cada criterio anatómico se resumen en el Cuadro No.2, donde la columna de técnica de alto kilovoltaje representa la fracción total de observadores que visualizaron mejor esta estructura en la placa de alto kilovoltaje (basado en las entradas de las columnas $(+2)$ y $(+1)$ del Cuadro No.1). La columna de igual porcentaje representa la fracción que no tiene preferencia particular por alguna de las 2 opciones (basado en la entrada de la columna (0) del Cuadro No.1) y 


\begin{tabular}{|c|c|c|c|c|c|c|}
\hline \multicolumn{7}{|c|}{$\begin{array}{c}\text { Cuadro } 1 \\
\text { Frecuencia de preferencias de los diferentes } \\
\text { hallazgos radiológicos según las } 2 \text { técnicas } \\
\text { radiográficas }\end{array}$} \\
\hline & \multicolumn{6}{|c|}{ Puntuación } \\
\hline & +2 & +1 & 0 & -1 & -2 & NA \\
\hline \multicolumn{7}{|l|}{ Arcos costales } \\
\hline posteriores & 30 & 41 & 39 & 126 & 72 & 0 \\
\hline Intersticio & 106 & 115 & 18 & 46 & 13 & 10 \\
\hline Vascularidad & 95 & 122 & 33 & 45 & 11 & 2 \\
\hline \multicolumn{7}{|l|}{ Reflexión pleurales } \\
\hline \multicolumn{7}{|l|}{ Area sub } \\
\hline diafragmática & 17 & 42 & 109 & 94 & 22 & 24 \\
\hline Area Retrocardiaca & 13 & 24 & 67 & 105 & 30 & 69 \\
\hline Tejidos blandos & 27 & 75 & 68 & 105 & 27 & 6 \\
\hline Laringe-tráquea & 22 & 102 & 51 & 89 & 41 & 3 \\
\hline Pleura & 22 & 78 & 76 & 76 & 15 & 41 \\
\hline Apex pulmonar & 43 & 97 & 44 & 97 & 24 & 3 \\
\hline \multicolumn{7}{|l|}{ Anormalidades } \\
\hline cardiacas & 20 & 50 & 82 & 36 & 10 & 110 \\
\hline \multicolumn{7}{|l|}{ Corazón y } \\
\hline Grandes vasos & 38 & 91 & 92 & 60 & 12 & 15 \\
\hline Calcificaciones & 17 & 40 & 28 & 89 & 28 & 106 \\
\hline Nódulos & 21 & 67 & 16 & 36 & 19 & 149 \\
\hline Patrón alveolar & 39 & 66 & 14 & 18 & 8 & 163 \\
\hline Derrame pleural & 9 & 13 & 19 & 21 & 6 & 240 \\
\hline
\end{tabular}

la columna de técnica de bajo kilovoltaje representa la fracción total de observadores que visualizaron mejor esta estructura en la placa de bajo kilovoltaje (basado en las entradas de las columnas (-2) y (-1) del Cuadro No.1). Las estructuras anatómicas que se observan con un porcentaje mayor al $50 \%$ en la técnica de al to kilovoltaje son: intersticio, vascularidad, reflecciones pleurales mediastinales, nódulos y patrón alveolar. Las estructuras anatómicas que se observan con un porcentaje mayor al $50 \%$ en la técnica de bajo kilovoltaje son: costillas, arcos posteriores, área retrocardiaco y calcificaciones.

\section{Discusión}

En la mayoría de los hospitales del sistema de seguridad social y en las clínicas privadas de Costa Rica, las placas PA de tórax se realizan con técnicas de bajo kilovoltaje; sin embargo, en el ámbito internacional se recomienda el uso de kilovoltajes altos. El Colegio Americano de Radiología utiliza kilovoltajes de 120 a 150 con técnica antidispersión (empleando parrilla Bucky o técnica de aire) 19,20 para mejorar la forma como se visualizan los objetos de bajo contraste y disminuir las dosis de radiación. Los resultados mostraron que más del $50 \%$ de los médicos prefirieron la técnica de alto $\mathrm{kV} p$, aunque no se encuentren familiarizados con imágenes de bajo contraste.

Dentro del marco de la protección radiológica dictada por los organismos internacionales, 12,13,14 las dosis máximas de entrada a la piel, con las cuales se obtiene un estudio de

\begin{tabular}{|c|c|c|c|}
\hline \multicolumn{4}{|c|}{$\begin{array}{c}\text { Cuadro } 2 \\
\text { Porcentaje de preferencia, según técnica } \\
\text { radiográfica para los diferentes hallazgos } \\
\text { radiológicos evaluados }\end{array}$} \\
\hline \multicolumn{4}{|c|}{ Porcentaje de preferencia } \\
\hline & $\begin{array}{l}\text { Técnica } \\
\text { alto } k V p\end{array}$ & Igual & $\begin{array}{r}\text { Técnica } \\
\text { bajo } \mathrm{kVp}\end{array}$ \\
\hline Costillas arcos posteriores & 23 & 13 & 64 \\
\hline Intersticio & 74 & 6 & 20 \\
\hline Vascularidad & 71 & 11 & 18 \\
\hline Reflexión pleurales mediastinales & 50 & 26 & 24 \\
\hline Sub diafragmático & 21 & 38 & 41 \\
\hline Retrocardiaco & 15 & 28 & 56 \\
\hline Tejidos blandos & 34 & 23 & 44 \\
\hline Laringe-traquea & 41 & 17 & 43 \\
\hline Pleura & 37 & 28 & 34 \\
\hline Apex pulmonar & 46 & 14 & 40 \\
\hline Anormalidades cardiacas & 35 & 41 & 23 \\
\hline Corazón y grandes vasos & 44 & 31 & 25 \\
\hline Calcificaciones & 28 & 14 & 58 \\
\hline Nódulos & 55 & 10 & 35 \\
\hline Patrón alveolar & 72 & 10 & 18 \\
\hline Derrame pleural & 32 & 28 & 40 \\
\hline \multicolumn{4}{|c|}{$\begin{array}{l}\text { Porcentajes de preferencia para cada hallazgo o patología evaluada. La colum } \\
\text { na "Técnica Alto kVp" corresponde al porcentaje total a las entradas de (+2) y } \\
(+1) \text { del cuadro No.1, la columna "Igual" corresponde al porcentaje de las } \\
\text { entradas de (0) del cuadro No.1 y la columna "Técnica Bajo kVp" corresponde } \\
\text { al porcentaje total de las entradas de (-2) y (-1) del cuadro No.1. }\end{array}$} \\
\hline
\end{tabular}

calidad satisfactoria (nivel orientativo de dosis), se alcanzan con la técnica propuesta, pues en un $100 \%$ de los pacientes se entregó una dosis por debajo del nivel recomendado, no siendo este el caso para las dosis entregadas con técnicas de bajos kilovoltajes, ya que en un $73 \%$ de los pacientes la dosis superó el nivel orientativo.

En protección radiológica se introduce la magnitud de dosis efectiva (mSv), que conceptualmente es disto de a la dosis absorbida (mGy), pues toma en cuenta los factores de riesgo radiobiológicos (el tipo de órgano que está siendo irradiado y el tipo de radiación que se está utilizando: alfa, beta, rayos $X$, etc.) para la inducción de cánceres fatales. Para estimar las dosis efectivas con base en una medición en la piel del paciente, se deben utilizar algoritmos matemáticos más complicados, como lo es el análisis estadístico de M onte Carlo, ${ }^{21}$ que estudia la entrega de energía por cada uno de los mill ones de fotones de Rayos $X$ que entran en el cuerpo, basado en diferentes maniquís que asemejan al hombre de referencia. A nálisis realizados por otros autores, 3,22 que emplaron la metodología de $\mathrm{M}$ onte $\mathrm{Carlo}$, han calculado las dosis efectivas para radiografías de tórax PA y han encontrado que el riesgo de inducción de cáncer se minimiza al aumentar la energía del haz de radiación, pues las dosis efectivas encontradas también disminuyen al aumentar el kilovoltaje.

El análisis de la valoración diagnóstica por parte de los 28 médicos radiólogos, permite mostrar al cuerpo médico nacional que las técnicas de alto $\mathrm{kV} p$ mejoran la imagen de los pulmones y el mediastino; los bajos kVp acentúan las 
costillas y el contorno del mediastino, y por lo tanto obstruyen la visualización de la vascularidad pulmonar, las marcas broncopulmonares, las estructuras dentro del mediastino y las áreas detrás de las costillas y el corazón. En vista del beneficio al paciente, dado por la disminución de la dosis individual y colectiva con las técnicas de altos kilovol tajes, y de que el médico indica que observa indistintamente ciertos hallazgos o patologías con ambas técnicas, bien se puede sugerir la suma de los porcentajes de preferencia de igual con ambas técnicas (columna central del Cuadro No.2), con el porcentaje de preferencia con la técnica de alto $\mathrm{kV} \mathrm{p}$ (columna izquierda del Cuadro No.2). Esto produce a que de los 16 parámetros val orados en la encuesta, ${ }^{13}$ resultaron mejor con la técnica de alto kilovoltaje. Solo los arcos costales posteriores, el área retrocardiaca y las calcificaciones se prefieren con técnicas de bajos kilovoltajes. De modo que, la técnica de al to kilovoltaje permite obtener más información diagnóstica (mejor visualización de las estructuras de bajo contraste) y disminuir las dosis a los pacientes.

Se concluye que en el proceso de optimización del estudio del tórax en busca de maximizar la razón riesgo versus beneficio, las técnicas empleadas en las radiografías de tórax en nuestro país deberán modificarse con base en las recomendaciones internacionales y en los resultados de este estudio.

El proceso de adaptación por parte del médico radiólogo y del médico tratante, a una placa menos contrastada, deberá ser un proceso paulatino ligado a capacitación, programas de control de calidad, ${ }^{23,24}$ actualización en protección radiológica y desarrollo tecnológico en el área de la radiología del tórax.

\section{Agradecimientos}

Se agradece a la Vicerrectoría de Investigación el apoyo brindado al proyecto N 0.112-A 0-003. A I Dr. F. Esquivel, por su ayuda en el diseño de la encuesta y en el análisis de las radiografías. A todos los médicos radiólogos que amablemente colaboraron en el análisis de la revisión de las radiografías: Dr. G. M eléndez, Dr. A. Duval, Dr. M. Soto, Dr. J. Barahona, Dr. R. B uján, Dr. M . Hernández, Dr. A. Escoto, Dr. Y. Salvatierra, Dr. R. Solís, D ra. M. Pacheco, Dr. J. Ulloa, Dr. F. Esquivel, Dra. C. Ponce, Dra. C. Odio, Dra. M. Salazar, Dra. L. Fonseca, Dr. J. Vargas, Dr. D. Sayago, Dr. F. M irambell, Dr. J. F. A nglada, Dr. J. R. Baltodano, Dr. J. M . Solera, Dra. G. M eléndez, Dra. M. Gómez, Dra. S. Núñez, Dra. A. Hernández, Dr. H. A bellán y Dr. M. Esquivel.

\footnotetext{
Abstract

Objective: Low kilovoltages ( $<100 \mathrm{kVp})$ to obtain chest films are commonly used in Costa Rica, regardless of international recommendations. This study aims to demonstrate that at higher $\mathrm{kV}$ ps the visualization of low contrast objects is improved and that the absorbed doses are lower.
}

M ethods: Two chests films, one at high $\mathrm{kV} p$ and one at low $k V p$, were taken to 11 patients. At the skin of each patient, two TLD-100 chips were placed to record the entrance skin doses, the chips were analyzed at the Nuclear Physics Laboratory of the University of Costa Rica. Using the anatomic criteria test tool 28 radiologists compared the anatomic findings for each technique.

Results: At higher $\mathrm{kV} p \mathrm{~s}$ the average entrance skin doses were lower by $40 \%$ and the overall contrast of the image was decreased between $10 \%$ to $50 \%$. The anatomic criteria test tool gave a total of 448 positive points, showing a clear preference for the high kilovoltaje technique.

Conclusions: The optimization of chest studies based on the ratio risk versus benefit shows that high kilovoltage techniques should be implemented in our country due to the diminution in the delivered doses and an improved visualization of the anatomy shown in the chest film according to the interpreting radiologists.

\section{Referencias}

1. Glazer HS, M uka E, Sagel SS, J ost RG. N ew techniques in chest radiography. Adv Chest Radiol 1994; 32: 711-729.

2. Proto AV, Lane EJ. $350 \mathrm{kV}$ chest radiography: review and comparison with 120 kV p. A m J Roentgenol 1978; 130: 859-866.

3. Pehrone T, Steidley D, A ppleley A, Christman E, Hanghey F. X-ray beam energy, scatter and radiation risk in chest radiography. Health Phys 1996; 70: 488-497.

4. Kelsey C. Techniques for chest radiography. En: Haus A. editor, A merican Institute of Physics, Inc., The physics of medical imaging: recording system measurements and techniques. N ew Y ork: A merican Institute of Physics 1979: 442-458.

5. Vucich J. The role of anatomic criteria in the evaluation of radiographic images En: Haus A . editor, A merican Institute of Physics, Inc., The physics of medical imaging: recording system measurements and techniques. New York: A merican Institute of Physics 1979: 573-587.

6. Jacobi $C$, Paris $D$. Manual de técnica radiológica. Buenos Aires: Editorial El A teneo Pedro García SA 1980.

7. Sprawls P. Physical principles of medical imaging. Wisconsin: M edical Physics Publishing 1995.

8. MacM ahon $\mathrm{H}$, Vyborny $\mathrm{C}$. Technical advances in chest radiography. AJR 1994; 163: 1049-1059.

9. Oda N, Nakata H, M urakami S, Terada K, Nakamura K, Yoshida A. Optimal beam quality for chest computed radiography. Invest Radiol 1996; 31: 126-131.

10. Wieder $\mathrm{S}, \mathrm{A}$ dams $\mathrm{P}$. Improved routine chest radiography with a trough filter. A m J Roentgenol 1981; 137: 695-698.

11. Mora P. Radiaciones recibidas por los pacientes en el Servicio de Radiología del Hospital Calderón Guardia. Acta Medica Costarric 1999; 41: 22-27.

12. Parry RA, Sharon AG, A rcher BR. The AAPM/RSNA physics tutorial for residents: typical patient radiation doses in diagnostic radiology. Imaging and therapeutic technology 1999; 19: 1289-1302.

13. Comisión Internacional de Protección Radiológica (CIPR). Recommendations of the ICRP Publication 60 (1990). International Commission on Radiological Protection. Nueva Y ork: Pergamon Press, 1991.

14. Organismo Internacional de Energía A tómica. Normas Básicas Internacionales de Seguridad para la Protección contra la Radiación Ionizante y la Seguridad de las Fuentes de Radiación, Colección de Seguridad N 0.115. Viena: OIEA, 1997. 
15. Wochos JF, Fullerton GD, DeW erd LA. M ailed Thermoluminescent Dosimeter Determination of Entrance Skin Exposure and Half-value Layer in M ammography. A m J Roentgenol 1978; 131: 617-619.

16. DeW erd LA, Chiu NB. The Determination of Radiation Dose by Mail for Diagnostic Radiological Examinations with Thermoluminescent Dosemeters. Rad Prot D osimetry 1993; 47: 509-512.

17. Herrou J, Bendu T, Campbell W, Sumkin J, Rockette H, Gur David. Effects of luminescence and resolution on observer performance with chest radiographs. Radiology 2000; 215: 169-174.

18. L. Romo. M étodos de Experimentación Científica. Ecuador: Editoria Universitaria 1973.

19. A merican College of Radiology. Standards for performance of adult chest radiography. Reston, Virginia: ACR, 1990.
20. K uhns LR, Kottamasu SR. Pediatric air-gap chest digital imaging: an experimental study. Pediatr Radiol 1995; 25: 199-201.

21. Y.A. Sherider, The M onte Carlo M ethod. Nueva Y ork: Pergamon Press 1966.

22. Schultz FW, Geleijns J, Zoetelief J. Calculation of dose conversion factors for posterior-anterior chest radiography of adults with a relatively high-energy X-ray spectrum. Br J Radiol 1994; 67: 775-785.

23. Ramirez G, A costa N, Cuenca R. Construction of a thorax phantom that permits the study of the conditions of image and physical parameters of an X-ray equipment. Physica M edica 1990; 6: 277-281.

24. Chotas HG, Floyd CE, Johnson GA, Ravin CE. Quality control phantom for digital chest radiography. Radiology 1997; 202: 111-116. 